Gynecologic and

Obstetric Investigation
Gynecol Obstet Invest 2018;83:105-115

DOI: $10.1159 / 000485619$
Received: April 19, 2017

Accepted after revision: November 21, 2017

Published online: December 19, 2017

\title{
Subclinical Hypothyroidism Impact on the Characteristics of Patients with Polycystic Ovary Syndrome. A Meta-Analysis of Observational Studies
}

\author{
Sebastião Freitas de Medeiros ${ }^{a}$, Matheus Antônio Souto de Medeiros $^{b}$ \\ Cinthia Marenza Ormond ${ }^{b}$ Jacklyne Silva Barbosa ${ }^{b}$ \\ Márcia Marly Winck Yamamoto ${ }^{b}$ \\ a Department of Gynecology and Obstetrics, Medical School, Federal University of Mato Grosso, Mato Grosso, Brazil; \\ bTropical Institute of Reproductive Medicine and Menopause, Cuiabá, Brazil
}

\section{Keywords}

Hyperandrogenism • Polycystic ovary syndrome .

Subclinical hypothyroidism · Thyroid dysfunction

Abstract

Background/Aims: Definitive polycystic ovary syndrome (PCOS) diagnosis should exclude thyroid dysfunctions. The purpose of the study is to examine the impact of subclinical hypothyroidism on the characteristics of PCOS patients. Methods: A meta-analysis of the published observational studies was conducted. Medline, Scopus, and Cochrane database search was performed to identify the studies that compared euthyroid PCOS and subclinical hypothyroidism (SCH)-PCOS patients. A total of 9 studies were selected, totalizing the inclusion of 1,537 euthyroid PCOS and $301 \mathrm{SCH}-$ PCOS. The data were expressed as raw mean difference and standard error, using the random-effects model. Heterogeneity among studies was examined using the Cochran's test (Q) and I ${ }^{2}$ statistics. Results: Anthropometrical parameters were similar in both groups. Total cholesterol (TC) and triglyceride (TG) were higher in SCH-PCOS ( $p=0.036$ and $p=$ 0.012 ). High-density lipoprotein cholesterol was lower in the SCH-PCOS group $(p=0.018)$. Fasting glucose was lower in euthyroid PCOS $(p=0.022)$. All androgen levels were similar in both group ( $p>0.05$ for all). Conclusion: TC, TG and fasting glucose were higher in SCH-PCOS patients. Because of the heterogeneity among studies, some summarized results should be interpreted with caution. Consistent data for future studies addressing PCOS diagnosis are provided.

(c) 2017 S. Karger AG, Base

\section{Introduction}

The most common clinical features of polycystic ovary syndrome (PCOS) include oligomenorrhea, amenorrhea, infertility, hirsutism, weight gain, central obesity, and acanthosis nigricans. Hormonally, it can present elevated luteinizing hormone (LH) levels with normal or slightly decreased levels of follicle-stimulating hormone, hyperandrogenemia, hyperinsulinemia, and normal levels of cortisol (F), prolactin (PRL), thyroid-stimulating hormone (TSH), thyroxine (T4), and free thyroxin (FT4) [1]. Low levels of sex hormone binding-globulin (SHBG) may represent a hyperinsulinemic status. Dyslipidemia, dysglycemia, and insulin resistance, frequents in PCOS patients, increase the

\section{KARGER}

(c) 2017 S. Karger AG, Basel

E-Mail karger@karger.com

www.karger.com/goi
Sebastião Freitas de Medeiros

Rua: Almirante Henrique

Pinheiro Guedes, 195, Bairro: Duque de Caxias

Cuiabá, MT CEP 78043-306 (Brazil)

E-Mail de.medeiros@ terra.com.br 
risks of type II diabetes mellitus and cardiovascular disease [2].

Primary overt hypothyroidism shares many of these signs and symptoms with PCOS. Clinically, patients with hypothyroidism may present menstrual disorders, infertility, hyperandrogenism signs, and weight gain [3]. Among the endocrine aspects, it can be characterized by mild increase in total testosterone $(\mathrm{T})$ and free testosterone (fT) levels, increased total and free estradiol (E2), high PRL and LH, and decreased SHBG levels [3]. Dyslipidemia and insulin resistance are also common in hypothyroidism [4]. Moreover, ovaries with multicystic appearance are frequently found in patients with such thyroid dysfunction [5]. It must be noted that ovaries with multicystic appearance can be seen in other conditions including hypothalamic amenorrhea and women who used combined oral contraceptive.

Most subjects with subclinical hypothyroidism $(\mathrm{SCH})$ have few symptoms of thyroid dysfunction, but it can lead to clinical, endocrine, or metabolic changes such as dyslipidemia, dysglycemia, insulin resistance, infertility, ovulatory dysfunction, obesity, and abnormal menstrual cycle $[3,6-8]$. High levels of T, fT, LH, PRL, fasting and postprandial insulin, and glycated hemoglobin (HbA1C), increased homeostasis model assessment of insulin resistance index (HOMA-IR), and decreased levels of SHBG may be present in $\mathrm{SCH}[5,9,10]$. Furthermore, $\mathrm{SCH}$ seems to be an independent risk factor for infarction and it is more frequent in women with metabolic syndrome $[9,11,12]$. Currently, well designed studies connecting or dissociating PCOS and SCH are still lacking [13].

Though definitive PCOS diagnosis should be confirmed only after exclusion of any other hyperandrogenic entity, including thyroid dysfunction [14], a number of studies have not described the criteria used to exclude hypothyroidism before confirming PCOS diagnosis [15-17] and many other studies have not measured, or reported TSH levels at all $[18,19]$. Among the studies that clearly reported to have excluded thyroid dysfunction before the definitive diagnosis of PCOS, the used criteria were extremely different: manifested hypothyroidism [20], clinical suspicion of hypothyroidism [21], and different levels of TSH, ranging from 2.5 to $5.0 \mu \mathrm{UI} /$ $\mathrm{mL}$ to consider euthyroidism $[4,12,22-28]$. Given the lack of a specific guideline on this matter and the need for clearly defining which thyroid dysfunctions should be excluded from PCOS diagnosis, the present study aimed to examine the available literature comparing the characteristics of euthyroid PCOS and PCOS patients presenting $\mathrm{SCH}$.

\section{Material and Methods}

The aim was to evaluate extant data regarding the characteristics of PCOS and SCH-PCOS patients. The electronic review of studies reporting on this matter was limited to English language. A combination of the terms PCOS and thyroid dysfunction, PCOS and $\mathrm{SCH}$, hyperandrogenism and thyroid function, and oligomenorrhea and hypothyroidism were searched in Medline, Scopus, and Cochrane database. Additionally, references in reviews or original articles were hand-searched. A total of 154 potential articles were found and 135 were excluded after browsing the titles and abstracts. A total of 19 full papers and a complete abstract were assessed for eligibility. All included studies used the current established criteria to diagnose PCOS. Later, 11 publications were excluded because they did not compare PCOS with SCH-PCOS head to head or did not provide sufficient data for analysis [15-17, 23, $25,28-33$ ]. Attempts were made to contact the authors for further information but no answer was obtained. Finally, 9 full papers providing sufficient data for analysis were selected $[4,8,11,12,22,26$, $27,34,35]$. All inclusions were based on a clear description of PCOS with euthyroidism and PCOS with SCH. Study eligibility was verified independently by 2 authors (S.F.M. and M.M.W.Y.) and the study quality was assessed as recommended for meta-analysis of observational studies [36].

\section{Statistical Analyses}

Because the outcomes were reported on a meaningful same scale, the meta-analysis of enrolled studies was performed using the raw mean difference (RMD) [37]. Anthropometric, metabolic, and hormonal characteristics were compared between euthyroid PCOS and SCH-PCOS patients. The random-effects model was chosen because the included studies were not functionally identical when they have used different TSH cut-off levels to define SCH and may have different effect sizes [38]. Between-studies variance of the effect size $\left(\tau^{2}\right)$ was estimated using the method of moments [39]. In addition, heterogeneity among studies was examined using Cochran's Q test and quantified by the proportion of the observed variance as a measure of the degree of inconsistency in the studies using $\mathrm{I}^{2}$ statistics $[38,39]$. All analyses were performed using the Comprehensive Meta-Analysis software, version 2 (Biostat Inc., NJ, USA).

\section{Results}

Data comparing euthyroid PCOS and SCH-PCOS patients were extracted from 9 articles. All were already published in peer-reviewed journals between 2009 and 2017. All included studies were observational (Table 1): 6 were cross-sectional $[4,22,26,27,34,35]$ and 3 were casecontrol $[8,11,12]$. A total of 1,837 patients were enrolled, 1,537 euthyroid PCOS and 301 SCH-PCOS. In order to define PCOS, 1 study used National Institutes of Health $(\mathrm{NIH})$ criteria [12], 1 used both $\mathrm{NIH}$ and Rotterdam criteria [4], and 7 used only Rotterdam criteria $[8,11,22,26$, $27,34,35]$. The TSH normal upper limit was set at 2.5 in 
Table 1. Characteristics of observational studies comparing euthyroid PCOS patients and PCOS patients with subclinical hypothyroidism

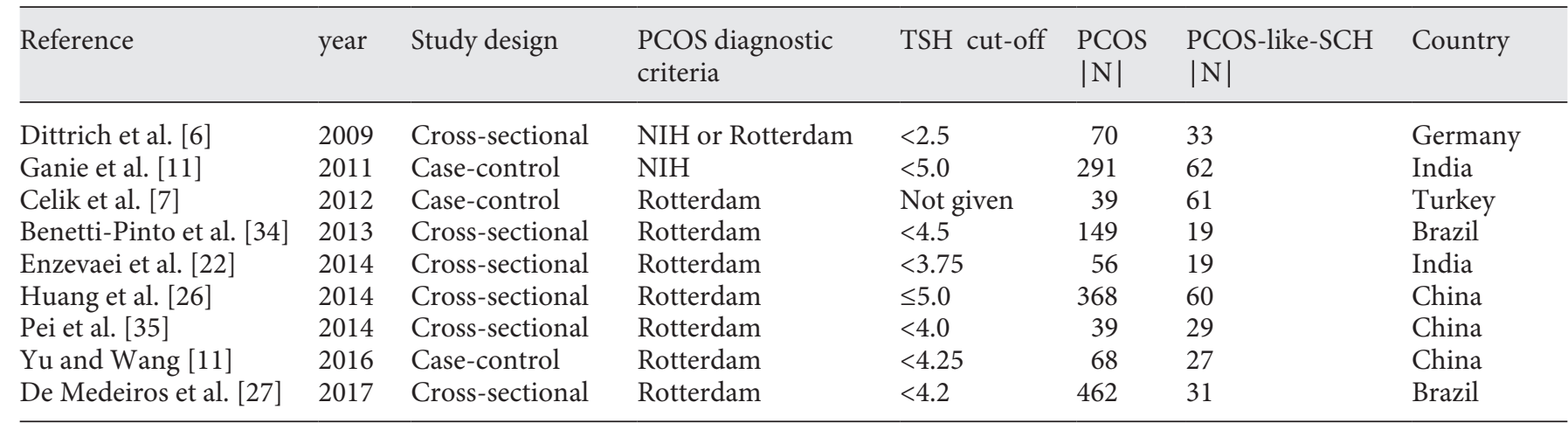

1 study [4], 3.75 in 1 study [22], 4.0 in 1 study [35], 4.2 in 1 study [27], 4.25 in 1 study [11], 4.5 in 1 study [32], and 5.0 in 2 studies $[12,26]$. One study did not provide a normal cut-off for TSH [8].

As a range of TSH cut-off levels was used in the included studies, the serum TSH concentrations were considerably different between euthyroid PCOS and SCHPCOS patients. All the 9 studies reported on TSH, including 1,537 euthyroid PCOS and 301 SCH-PCOS patients, and the raw mean difference (RMD) was $-4.193, \mathrm{SE}=$ $0.404(p<0.001)$. Due to the different cut-off used for $\mathrm{TSH}$, these studies were highly heterogeneous regarding this aspect $\left(\tau^{2}=1.216, \mathrm{Q}=264.66, \mathrm{I}^{2}=96.97 \%, p<0.001\right)$. FT4 concentrations were similar in euthyroid PCOS and SCH-PCOS patients (1,113 PCOS and 221 SCH-PCOS, $\mathrm{RMD}=0.173, \mathrm{SE}=0.386, p=0.654)$ in 7 heterogeneous studies $\left(\tau^{2}=0.578, \mathrm{Q}=18.21, \mathrm{I}^{2}=67.05 \%, p=0.006\right)$.

The ages of euthyroid PCOS patients and SCH-PCOS patients were not different and the summarized effect size of 9 enrolled studies was $\mathrm{RMD}=-0.467, \mathrm{SE}=0.550(p=$ $0.396)$. The studies showed moderate heterogeneity in their effect size $\left(\tau^{2}=1.519, \mathrm{Q}=19.17, \mathrm{I}^{2}=58.27 \%, p=\right.$ $0.014)$. A summary comparing anthropometric data between the 2 groups is shown in Figure 1. Waist circunferences were similar between euthyroid PCOS and SCHPCOS patients (958 PCOS and $131 \mathrm{SCH}-\mathrm{PCOS}, \mathrm{RMD}=$ $-0.517, p=0.671)$; the data were obtained from 4 homogeneous studies $\left(\tau^{2}=0.000, p=0.633\right)$. The waist to hip ratios (WHR) also were not different in euthyroid PCOS and SCH-PCOS patients (1,216 euthyroid PCOS and 192 SCH-PCOS patients, $\mathrm{RMD}=-0.004, p=0.597$ ) according 5 homogeneous studies $\left(\tau^{2}=0.000, p=0.582\right)$. Body mass indexes (BMIs) were similar in euthyroid PCOS and SCH-PCOS patients (1,573 PCOS and $300 \mathrm{SCH}-\mathrm{PCOS}$, $\mathrm{RMD}=-0.613, p=0.237$ ); the 9 studies reporting on this

Subclinical Hypothyroidism Impact on PCOS variable presented moderate homogeneity $\left(\tau^{2}=1.039, p=\right.$ 0.051).

Comparisons of the different lipid concentrations between euthyroid PCOS and SCH-PCOS women are given in Figure 2. Total cholesterol (TC) levels were lower in euthyroid PCOS when compared with SCH-PCOS patients in 9 studies (1,537 PCOS and $300 \mathrm{SCH}-\mathrm{PCOS}$ patients, $\mathrm{RMD}=-0.395, p=0.010)$, but the studies were heterogeneous regarding this parameter $\left(\tau^{2}=0.164, p<\right.$ 0.001 ). High density lipoprotein cholesterol (HDL-C) levels were higher in euthyroid PCOS patients $(1,285$ PCOS and 271 SCH-PCOS patients, RMD $=0.100, p=$ $0.004)$, summary of 8 slightly heterogeneous in 8 studies $\left(\tau^{2}=0.004, p=0.065\right)$. Low density lipoprotein cholesterol (LDL-C) levels were not different in euthyroid PCOS and SCH-PCOS patients (1,285 PCOS and $271 \mathrm{SCH}-$ PCOS patients, RMD $=-0.100, p=0.384)$ and the 8 studies had heterogeneous effect size $\left(\tau^{2}=0.086, p<0.001\right)$. Triglyceride (TG) levels were higher in SCH-PCOS patients $(1,537$ PCOS and 300 SCH-PCOS patients, RMD = $-0.281, p=0.002)$, according 9 heterogeneous studies $\left(\tau^{2}=0.048, p<0.001\right)$.

Comparisons of the carbohydrate metabolism mediators between euthyroid PCOS and SCH-PCOS patients are given in Figure 3. Fasting glucose concentrations were significantly higher in SCH-PCOS patients when compared with euthyroid PCOS patients (1,469 PCOS and $273 \mathrm{SCH}-\mathrm{PCOS}$ patients, $\mathrm{RMD}=-0.109, p=0.022)$; the 8 included studies were highly homogeneous $\left(\tau^{2}=0.001\right.$, $p=0.022$ ). Fasting insulin levels were not different in SCH-PCOS and euthyroid PCOS patients (1,101 PCOS and 213 SCH-PCOS patients, RMD $=-0.180, p=0.176$ ); the 7 studies presented moderate and significant heterogeneity $\left(\tau^{2}=0.071, p=0.021\right)$. HOMA-IR was not different in PCOS and SCH-PCOS patients (1,537 PCOS and 


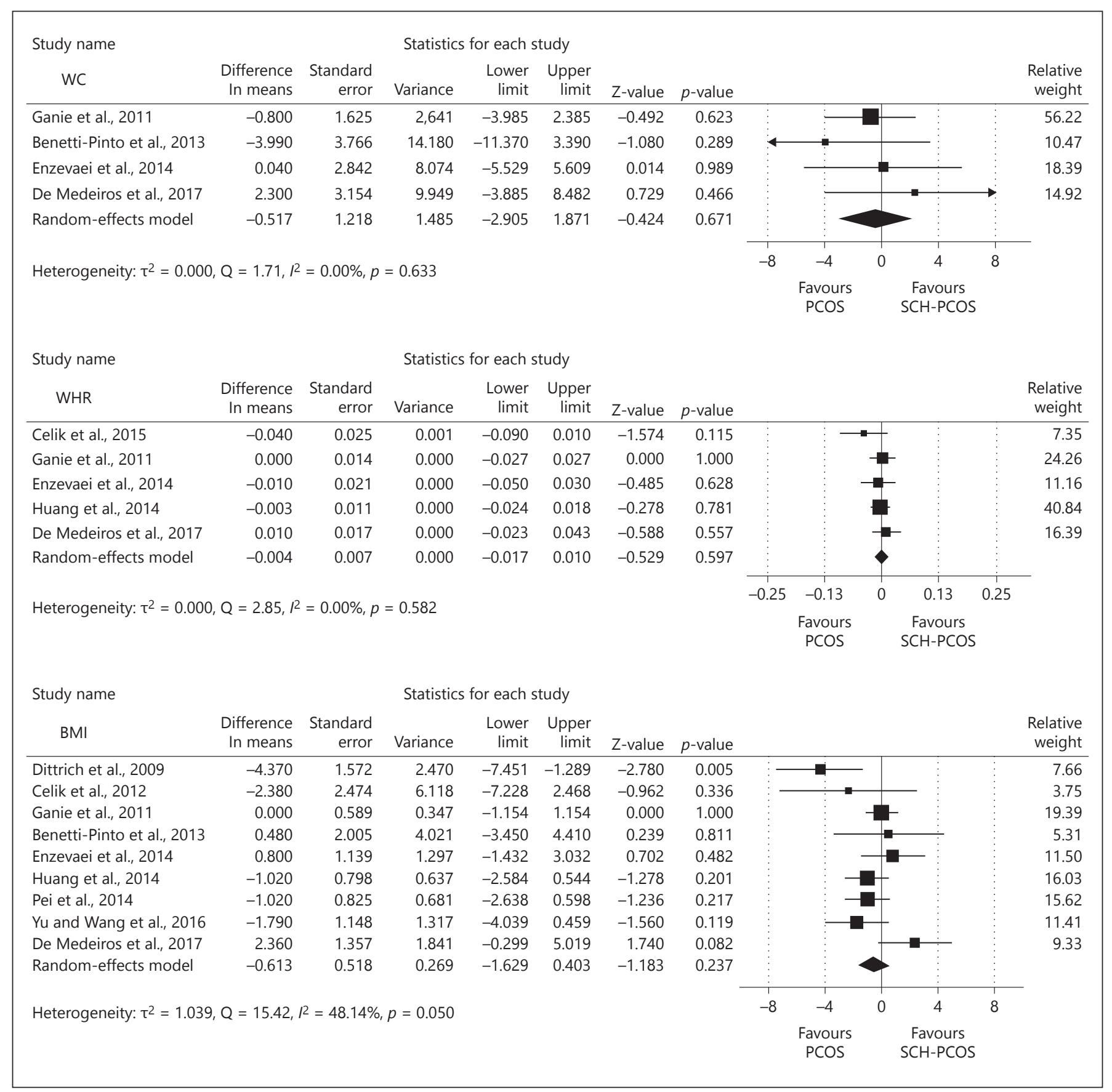

Fig. 1. Forest plot of the raw difference means of anthropometric parameters between euthyroid-PCOS and SCH-PCOS patients.

300 SCH-PCOS patients, RMD $=-0.163, p=0.616)$ but the heterogeneity among studies was high $\left(\tau^{2}=0.758, p<\right.$ $0.001)$.

The summary of the differences in androgen concentrations between euthyroid PCOS and SCH-PCOS patients is shown in Figure 4. Total $\mathrm{T}$ concentrations were similar in euthyroid PCOS and SCH-PCOS (1,379 PCOS and $266 \mathrm{SCH}$-PCOS patients, RMD $=-0.114, p=0.676)$; the 6 studies presented high heterogeneity $\left(\tau^{2}=0.408, p<\right.$ 0.001 ). The levels of $\mathrm{fT}$ also were not different in euthyroid PCOS and SCH-PCOS patients (754 PCOS and 96 $\mathrm{SCH}-\mathrm{PCOS}$ patients, $\mathrm{RMD}=0.001, p=0.916)$ in 4 het- 


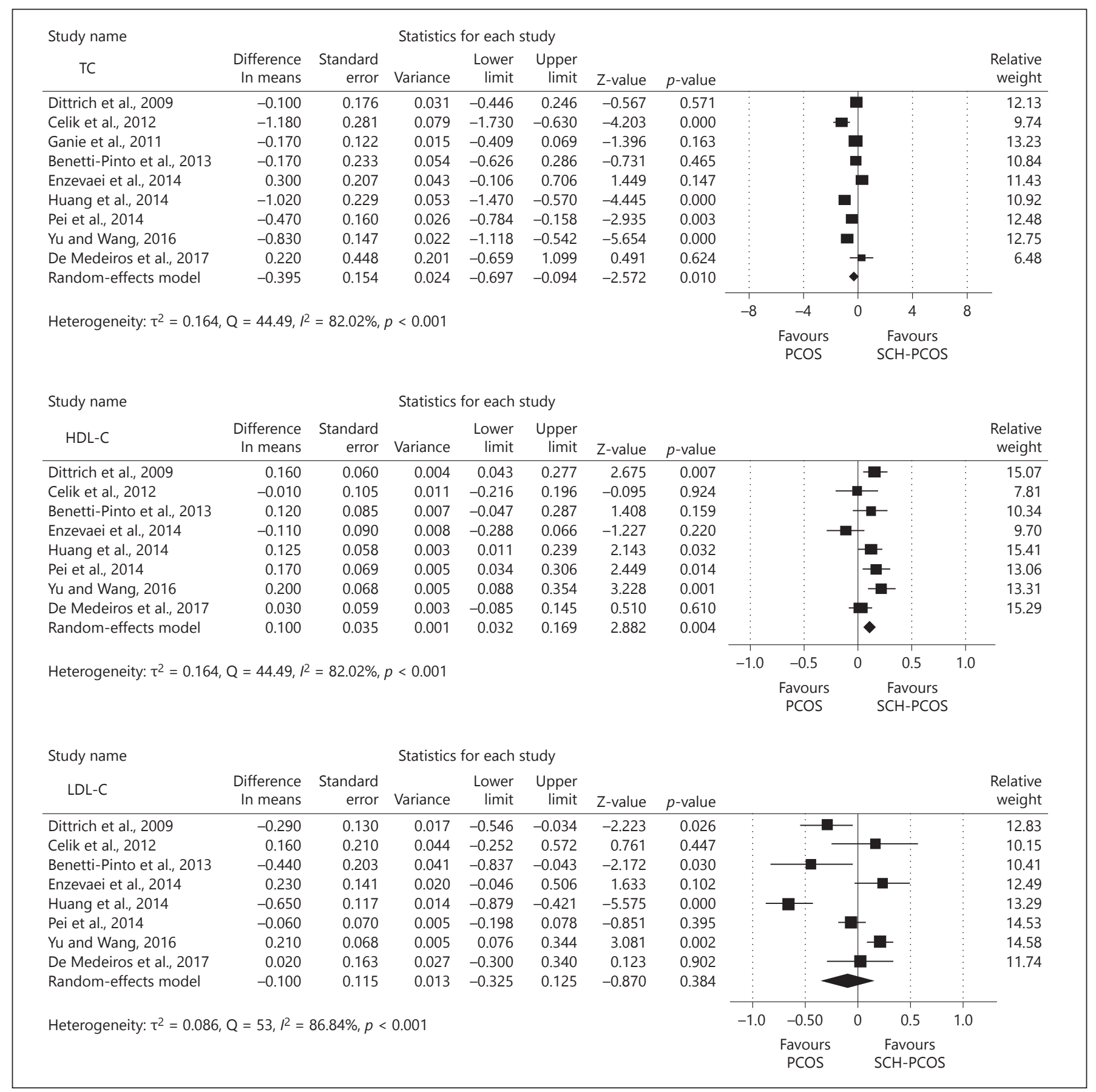

Fig. 2. Forest plot of the lipids'raw difference means between euthyroid-PCOS and SCH-PCOS patients.

(Figure 2 continued on next page.)

erogeneous studies $\left(\tau^{2}=0.000, p=0.009\right)$. SHBG concentrations also were not different when euthyroid PCOS patients were compared with SCH-PCOS patients $(900$ PCOS and 161 SCH-PCOS patients, RMD $=-8.877, p=$ $0.504)$; the 3 studies were highly heterogeneous $\left(\tau^{2}=\right.$ $512.67, p<0.001)$. Free androgen index was similar in
PCOS and SCH-PCOS patients (968 PCOS and $151 \mathrm{SCH}-$ PCOS patients, RMD $=-1.065, p=0.550)$; the 4 studies reporting on this parameter also were highly heterogeneous $\left(\tau^{2}=8.021, p=0.001\right)$. Dehydroroepiandrosterone sulfate (DHEAS) levels were similar in euthyroid PCOS and SCH-PCOS patients (1,049 PCOS and $143 \mathrm{SCH}-$ 


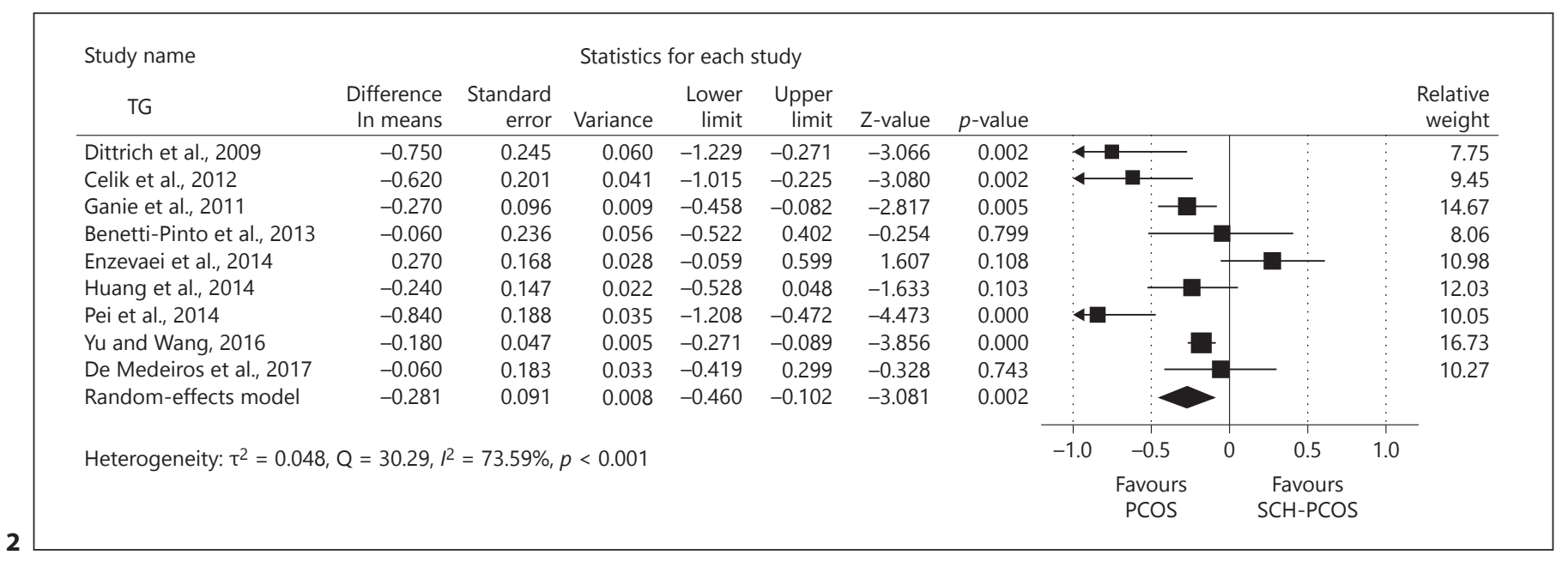

PCOS, RMD $=-0.571, p=0.315)$; the 4 studies also were highly heterogeneous regarding this parameter $\left(\tau^{2}=\right.$ $0.996, p=0.003)$.

\section{Discussion}

In view of the different criteria used to diagnose $\mathrm{SCH}$ from PCOS patients in the current literature, this metaanalysis was justified. The raw difference in mean ages of PCOS and SCH-PCOS patients verified in 9 studies with moderate heterogeneity was not statistically different. The meta-analysis also demonstrated that there were no difference between euthyroid PCOS and SCH-PCOS regarding some anthropometric parameters. However, 2 studies included in the meta-analysis showed higher BMI $[4,8]$ and another showed higher WHR [8] in SCH-PCOS patients when compared with euthyroid PCOS women. In previous studies, PCOS patients with $\mathrm{TSH} \geq 2.5 \mu \mathrm{UI} /$ $\mathrm{mL}$ and non-PCOS patients with high-normal serum TSH levels have shown visceral obesity $[8,40]$. A recent meta-analysis comparing SCH-PCOS with healthy control did not show any difference in anthropometric parameters between groups [41]. In general, the possible association between TSH levels and anthropometric parameters, mainly BMI, is still debatable and additional prospective studies should be undertaken to clarify the impact of elevated TSH levels on body shape. Comparisons of anthropometric parameters between $\mathrm{SCH}-\mathrm{PCOS}$ and normal controls have already shown similar results [25].

The comparisons of serum lipids between euthyroid PCOS and SCH-PCOS may have not provided a defini- tive conclusion in this meta-analysis because the heterogeneity among studies. However, the higher levels of TC in SCH-PCOS patients suggest that hypothyroidism may increase intestinal cholesterol absorption, overweighing the effects of decreased hepatic cholesterol synthesis [12, 15]. Increased TC level in non-PCOS women with $\mathrm{SCH}$ was already demonstrated in most, but not in all, studies [10,42]. Equally higher levels of TC were reported in PCOS patients with TSH either $>2.5$ or $<2.5$ [40], but a positive correlation between TSH and TC levels was reported even in euthyroid non-PCOS subjects [43]. Clearly, the results of the current metaanalysis indicate that TC concentrations are higher in $\mathrm{SCH}-\mathrm{PCOS}$ patients but because of the heterogeneity of the studies, this conclusion has to be taken with some cautions.

The finding of lower HDL-C levels in SCH-PCOS than in euthyroid PCOS patients in this meta-analysis supports the results of a previous study comparing euthyroid PCOS patients with TSH levels $>2.5$ or TSH $<2.5 \mu \mathrm{UI} / \mathrm{mL}$ [40]. In a non-PCOS population with SCH, the HDL-C level was found to be either similar or lower than the levels seen in normal controls. Further, no association between TSH and HDL-C concentrations was demonstrated in a recent study including non-PCOS individuals [44]. LDL-C levels were similar in euthyroid PCOS and $\mathrm{SCH}-\mathrm{PCOS}$ patients in the present meta-analysis. Similar levels of LDL-C in euthyroid PCOS women when compared with healthy controls were also reported [40]. In non-PCOS patients with hypothyroidism, LDL-C concentrations have been found to be either similar to [45] or higher than $[9,44]$ controls. The TG levels were significantly higher in SCH-PCOS patients than in euthyroid 


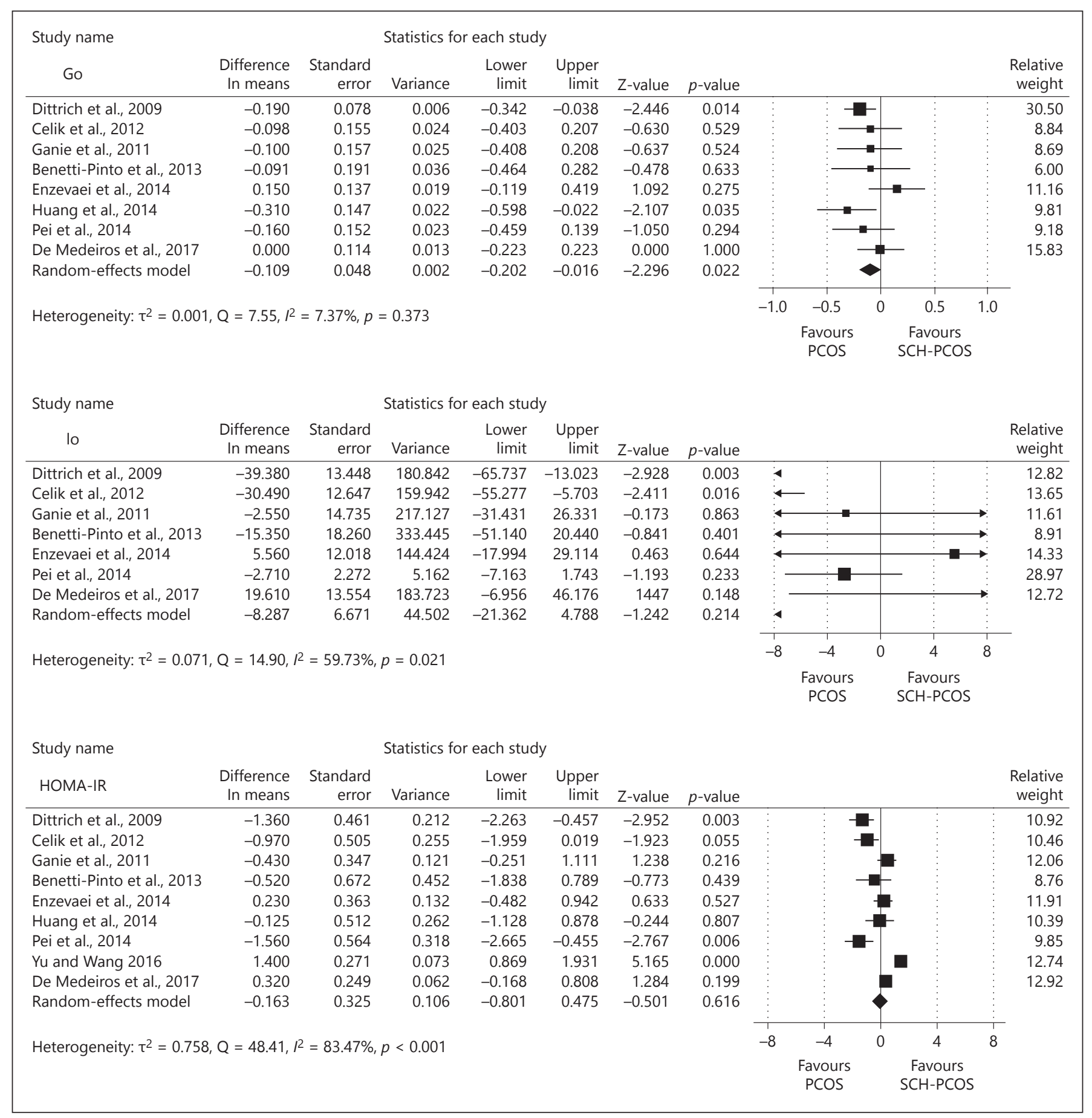

Fig. 3. Forest plot of the carbohydrates' raw difference means between euthyroid-PCOS and SCH-PCOS patients.

PCOS patients; previously higher levels of TG were reported in PCOS patients as a whole group [44]. In nonPCOS patients with SCH, TG levels were also found to be higher or not different from those found in healthy controls $[10,44,46]$.

Subclinical Hypothyroidism Impact on PCOS
Thyroid hormones may also modulate the carbohydrate metabolism and a possible association between TSH and fasting glucose levels was previously reported [10]. It was also reported that metformin usage results in slight reduction of TSH levels [47]. The current meta- 


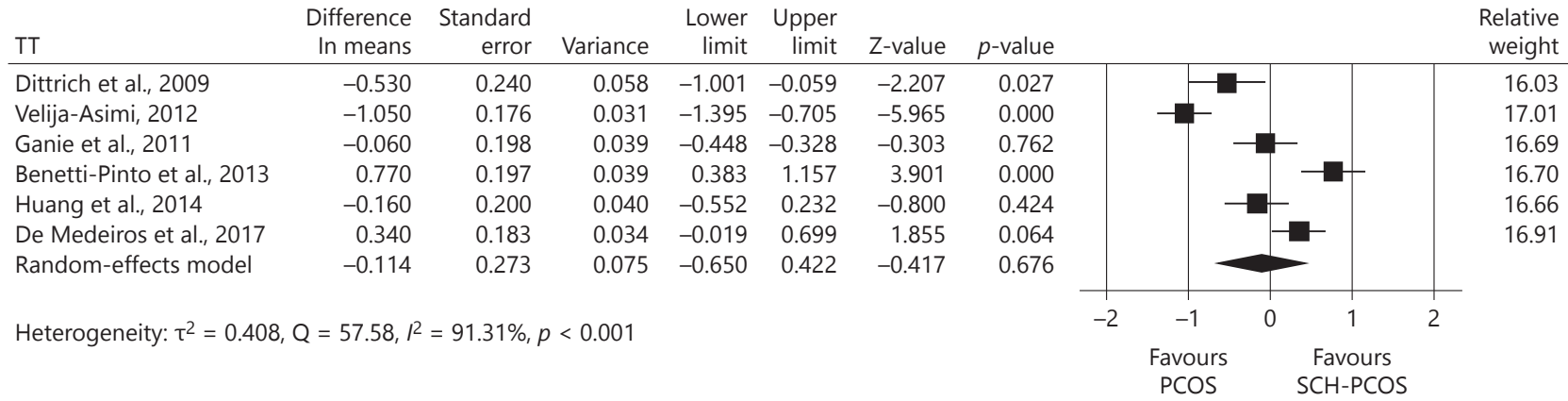

Study name

Statistics for each study

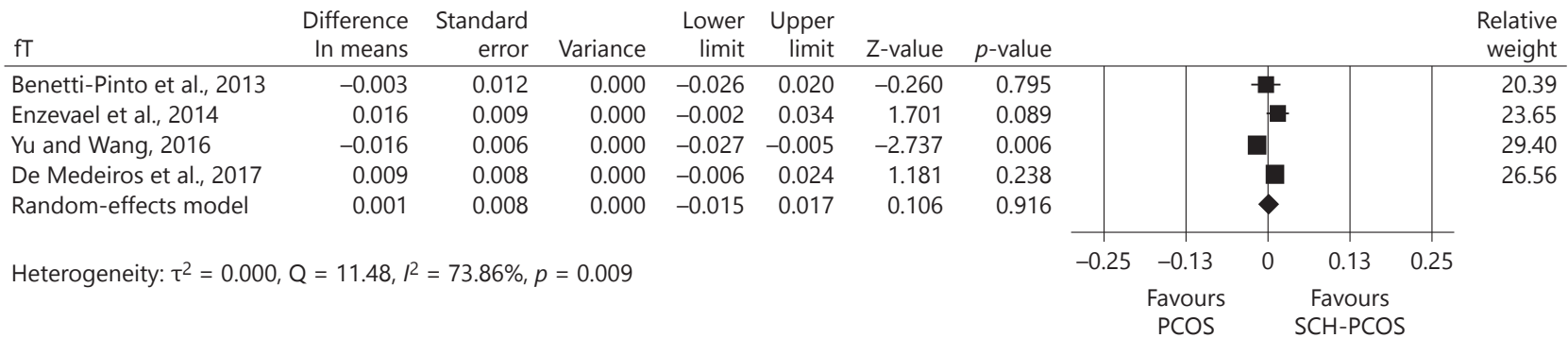

Study name

Statistics for each study

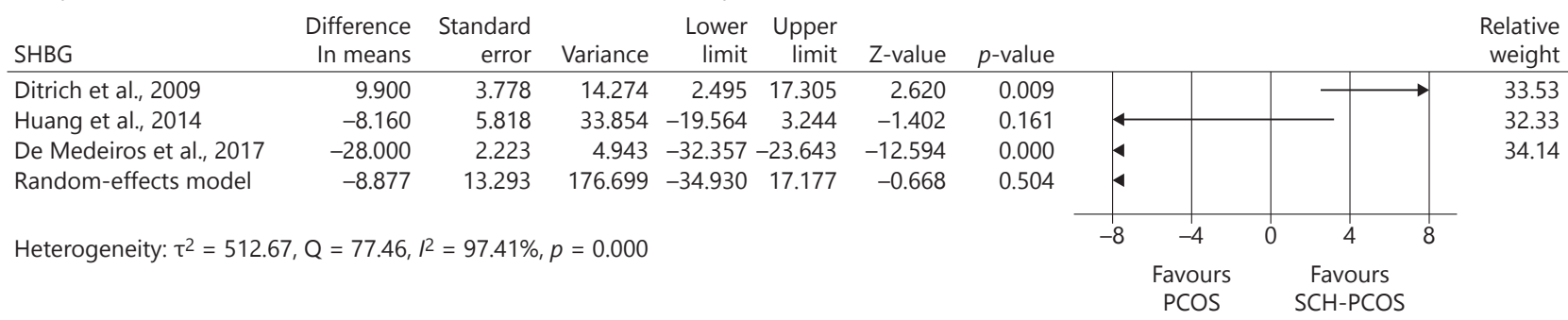

Study name

Statistics for each study

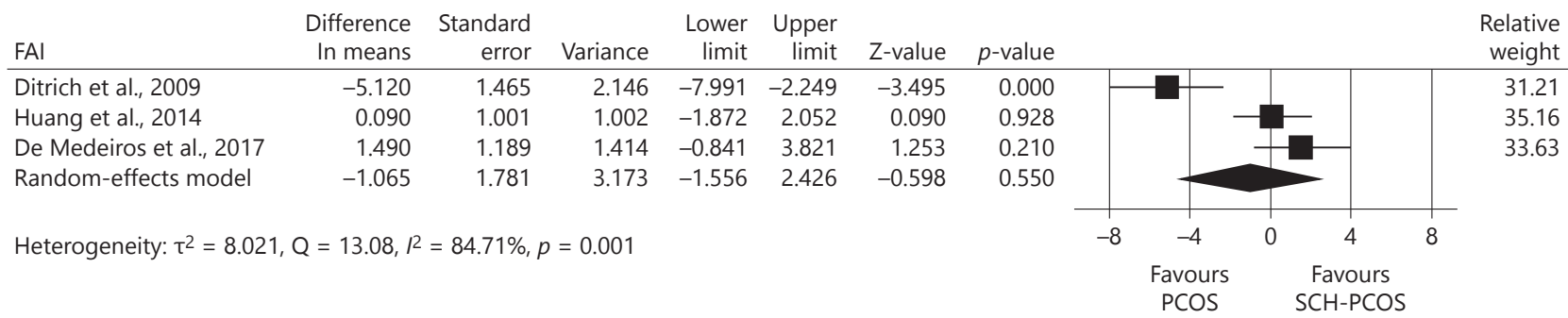

Fig. 4. Forest plot of the androgens' raw difference means between euthyroid-PCOS and SCH-PCOS patients.

(Figure 4 continued on next page.)

analysis demonstrated higher levels of fasting glucose in $\mathrm{SCH}-\mathrm{PCOS}$. Similar results have been found in 2 previous studies including PCOS patients $[4,26]$. In addition, PCOS patients as a whole group and healthy controls have shown similar fasting glucose levels in another study
[48]. Among non-PCOS patients, those with subclinical or overt hypothyroidism have presented similar [49] or higher [50] fasting glucose concentrations. The fasting insulin concentrations tended to be higher in $\mathrm{SCH}-\mathrm{PCOS}$ in this meta-analysis. In 2 of the included studies [7, 21], 


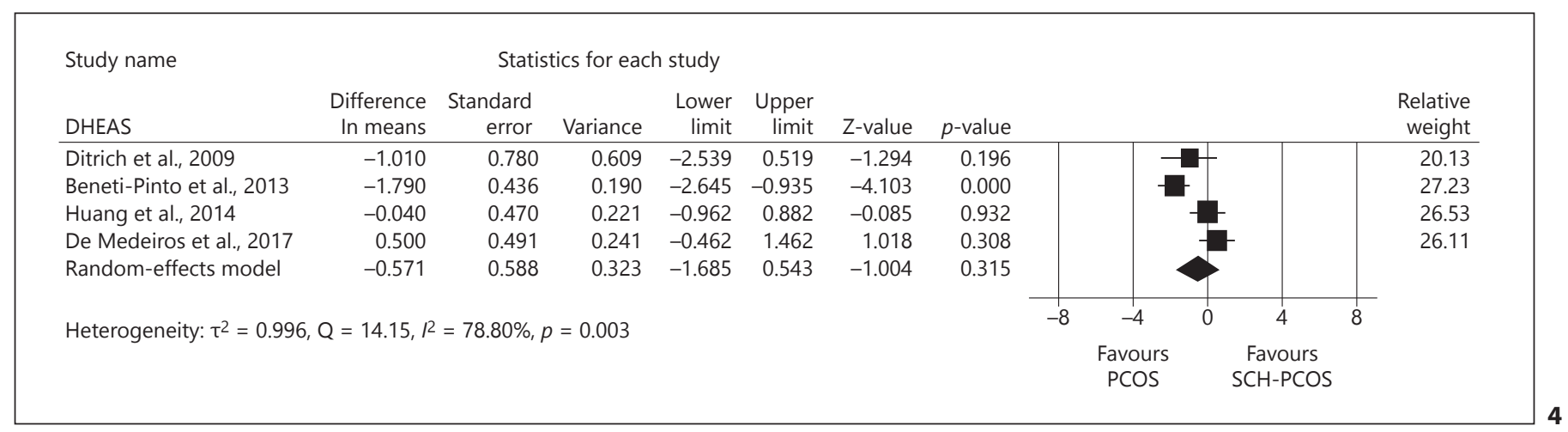

SCH-PCOS patients showed significantly higher fasting insulin levels. It was previously shown that patients with $\mathrm{SCH}$, either with or without PCOS, may present decreased insulin sensitivity in adipose and muscle tissues and higher levels of fasting insulin $[4,10,23]$. Some data have suggested that a decreased blood flow in peripheral tissues is the pathogenic mechanism behind increased insulin levels in women with hypothyroidism. The similar HOMA-IR in PCOS and SCH-PCOS patients demonstrated in the current meta-analysis was not consistent with the higher HOMA-IR previously reported in PCOS as a whole group when it was compared with healthy controls $[40,48]$ or non-PCOS patients with SCH [5]. A recent meta-analysis reported a significant decrease of HOMA-IR in SCH-PCOS and euthyroid PCOS as a whole group [41].

The current meta-analysis showed similar androgen concentrations in PCOS and SCH-PCOS patients. Only in 2, among 6 studies, total $\mathrm{T}$ levels were significantly higher in SCH-PCOS patients $[4,35]$. Increased T levels were also found in patients with $\mathrm{SCH}$ who had only PCOS morphology on ultrasonography [6]. In nonPCOS women, overt hypothyroidism status has been associated with low T levels [3], but the presence of anti-thyroid antibodies seems not to influence $\mathrm{T}$ concentrations [29]. Levels of fT have also been reported not to be different between SCH-PCOS and PCOS women $[22,27,34]$. In other study, patients with hypothyroidism were reported to have high $\mathrm{fT}$ levels as a consequence of lower SHBG hepatic production [6], but this meta-analysis and other publications have shown that SHBG levels were similar in PCOS and SCH-PCOS patients $[4,26,27]$. Individually, only the study by Dittrich et al. [6] reported significant reduction in SHBG in SCH-PCOS patients [4]. Based on 4 heterogeneous studies that have reported on DHEAS concentrations,
DHEAS levels were similar in PCOS and SCH-PCOS patients $[4,26,27,34]$. Only one study included in the meta-analysis reported significantly higher DHEAS concentrations in SCH-PCOS [34]. In other study, not included in this meta-analysis, DHEAS levels were not different in PCOS patients with or without hypothyroidism [51].

Although this meta-analysis tried to answer an interesting question, some limitations must be recognized: (a) instead of using a single cut-off, the enrolled and selected studies used a range from 2.5 to $5.0 \mu \mathrm{UI} / \mathrm{mL}$ as upper normal limit for TSH to exclude the diagnosis of hypothyroidism. Even the knowledge that iodine diet may influence the baseline TSH levels [52], in clinical practice it is essential to define a single cut-off for an uniform and definitive diagnosis of $\mathrm{SCH}$, (b) many of the included studies presented significant heterogeneity regarding the size effect of some biochemical and hormonal parameters, (c) the unequal sample size may have reduced the power of some findings. On the other hand, the current findings have scientific strength. One of the strengths of the study is the large number of patients included in the analysis. In addition, the meta-analysis highlights the need for establishing a single cut-off for TSH level in PCOS diagnosis and gives support for future studies regarding the impact of SCH on endocrine and metabolic changes in PCOS women.

\section{Conclusion}

In summary, though this meta-analysis showed that PCOS and SCH-PCOS share most endocrine and metabolic characteristics, a few differences must be considered. It was demonstrated that anthropometric parameters in PCOS and SCH-PCOS patients are similar. 
Higher TC, TG, and fasting glucose concentrations in SCH-PCOS than in euthyroid PCOS patients suggest that $\mathrm{SCH}$ may worsen the lipid and carbohydrate metabolism in PCOS. HDL-C is much lower in SCHPCOS than in euthyroid PCOS, attenuating the protective effect of HDL-C in the cardiovascular system of these patients. The differences in fasting glucose levels and some lipids need more long-term studies to establish their clinical implications. All summarized raw differences in the androgens mean levels showed no difference between PCOS and SCH-PCOS patients regarding these sex hormones. The clinical implications of the differences found between euthyroid PCOS and
SCH-PCOS are still unclear and more long-term studies with control of possible confounding covariates are yet required.

\section{Ethical Approval}

By this design (meta-analysis of published papers) an ethical approval is not necessary.

\section{Disclosure Statement}

The authors declare no conflict of interest.

\section{References}

1 de Medeiros SF, Barbosa JS, Yamamoto MM: Comparison of steroidogenic pathways among normoandrogenic and hyperandrogenic polycystic ovary syndrome patients and normal cycling women. J Obstet Gynaecol Res 2015;41:254-263.

2 Pasquali R, Patton L, Pagotto U, Gambineri A: Metabolic alterations and cardiovascular risk factors in the polycystic ovary syndrome. Min Ginecol 2005;57:79-85.

3 Krassas GE, Pontikides N, Kaltsas T, Papadopoulou P, Paunkovic J, Paunkovic N, et al: Disturbances of menstruation in hypothyroidism. Clin Endocrinol 1999;50:655-659.

4 Maratou E, Hadjidakis DJ, Kollias A, Tsegka K, Peppa M, Alevizaki M, et al: Studies of insulin resistance in patients with clinical and subclinical hypothyroidism. Europ J Endocrinol 2009; 160:785-790.

5 Ghosh S, Kabir SN, Pakrashi A, Chatterjee S, Chakravarty B: Subclinical hypothyroidism: a determinant of polycystic ovary syndrome. Horm Res 1993;39:61-66.

6 Dittrich R, Kajaia N, Cupisti S, Hoffman I, Beckmann MW, Mueller A: Association of thyroid-stimulating hormone with insulin resistance and androgen parameters in women with PCOS. Reprod Biomed Online 2009;19: 319-325.

7 Celik C, Abali R, Tasdemir N, Guzel S, Yuksel A, Aksu E, et al: Is subclinical hypothyroidism contributing dyslipidemia and insulin resistance in women with polycystic ovary syndrome? Gynecol Endocrinol 2012;28:615618.

8 Pearce EN: Update in lipid alterations in subclinical hypothyroidism. J Clin Endocrinol Metab 2012;97:326-333.

9 Bilic-Komarica E, Beciragic A, Junuzovic D: The importance of $\mathrm{HbA} 1 \mathrm{C}$ control in patients with subclinical hypothyroidism. Mat Soc Med 2012;24:212-219.

$10 \mathrm{Yu}$ Q, Wang JB: Subclinical hypothyroidism in PCOS: impact on presentation, insulin re- sistance, and cardiovascular risk. Biomed Res Int 2016;2016:2067087.

11 Ganie MA, Laway BA, Wani TA, Zargar MA,Nisar S,Ahamed F, et al: Association of subclinical hypothyroidism and phenotype, insulin resistance, and lipid parameters in young women with polycystic ovary syndrome. Fertil Steril 2011;95:20392043.

12 Uzunlulu M, Yorulmaz E, Oguz A: Prevalence of subliclinical hypothyroidism in patients with metabolic syndrome. Endocr J 2007;54: 71-76.

13 Singla R, Gupta Y, Khemani M, Aggarwal S: Thyroid disorders and polycystic ovary syndrome: an emerging relationship. Indian J Endocrinol Metab 2015;19:25-29.

14 Legro RS, Arslanian AS, Ehrmann DA, Hoeger KM, Murad MH, Pasquali R, et al: Diagnosis and treatment of polycystic ovary syndrome: an endocrine society clinical practice guideline. J Clin Endocrinol Metab 2013; 98:4565-4592.

15 El-Hafez HA, Elrakhawy MM, El-Aziz SA, ElEshmawy MM: Thyroid function and volume are associated with anthropometric measurements and insulin resistance in Egyptian women with polycystic ovary syndrome. J Diabetes Metab 2013;4:1-5.

16 Kachuei M, Fatemeh J, Kachuei, Keshteli AH: Prevalence of autoimmune thyroiditis in patients with polycystic ovary syndrome. Arch Gynecol Obstet 2012;285:853-856.

17 Mueller A, Schofl C, Dittrich R, Cupisti S, Oppelt PG, Schild RL, et al: Thyroid stimulating hormone is associated with insulin resistance independently of body mass index and age in women polycystic ovary syndrome. Hum Reprod 2009;24:2924-2930.

18 Yilmaz N, Kanat-Pektas M, Tongue E, Kilik S, Gulerman C, Gungor T, et al: The correlation of plasma homocysteine with insulin resistance in polycystic ovary syndrome. J Obstet Gynaecol Res 2008;34:384-391.
19 Broekmans FJ, Knauff EA, Valkenburg O, Laven JS, Eijkemans MJ, Fauser BC: PCOS according the Rotterdam consensus criteria: change in prevalence among WHO-II anovulation and association with metabolic factors. Brit J Obstet Gynaecol 2006;113: 1210-1217.

20 Azziz R, Woods KS, Reyna R, Key TJ, Knochenhauer ES, Yildiz BO: The prevalence and features of the polycystic ovary syndrome in an unselected population. J Clin Endocrinol Metab. 2004;89:2745-2749.

21 Carmina E, Rosato F, Janni A, Rizzo M, Longo RA. Relative prevalence of 387 different androgen excess disorders in 950 women referred because of clinical hyperandrogenism. J Clin Endocrinol Metab 2006;91:2-6.

22 Enzevaei A, Salehpour S, Tohidi M, Saharkhiz N: Subclinical hypothyroidism and insulin resistance in polycystic ovary syndrome: is there a relationship? Iran J Reprod Med 2014; 12:481-486.

23 Rotondi M, Cappelli C, Magri F, Botta R, Dionisio R, Iacobello $\mathrm{C}$, et al: Thyroidal effect of metformin treatment in patients with polycystic ovary syndrome. Clin Endocrinol 2011; 75:378-381.

24 Hamilton TE, Davis S, Onstad L, Kopecky KJ: Thyrotropin levels in a population with no clinical, autoantibody, or ultrasonographic evidence of thyroid disease: implications for the diagnosis of subclinical hypothyroidism. J Clin Endocrinol Metab 2008;93:1224-1230.

25 Sinha U, Sinharay K, Saha S, Longkumer A, Baul SN, Pal SK: Thyroid disorders in polycystic ovarian syndrome subjects: a tertiaty hospital based cross-sectional study from Eastern India. Ind J Endocrinol Metab 2013; 17:304-309.

26 Huang R, Zheng J, Li S, Tao T, Liu W: Subclinical hypothyroidism in patients with polycystic ovary syndrome: distribution and its association with lipid profiles. Eur J Obstet Gynecol Reprod Biol 2014;177:52-56. 
27 de Medeiros SF, Yamamoto MM, de Medeiros MS, Barbosa JS, Norman, RJ: Should subclinical hypothyroidism be an exclusion criterion for the diagnosis of polycystic ovary syndrome? J Reprod Infertil 2017;18:242250.

28 Iftikar R: The miracle of iodine complex in treatment of cases of polycystic ovarian disease induced by subclinical hypothyroidism. Isra Med J 2013;5:185-188.

29 Janssen OE, Mehlmauer N, Hahn S, Offner AH, Gartner R: High prevalence of autoimmune thyroiditis in patients with polycystic ovary syndrome. Eur J Endocrinol 2004;150: 363-369.

30 Morteza Taghavi S, Rokni H, Fatemi S: Metformin decreases thyrotropin in overweight women with polycystic ovarian syndrome and hypothyroidism. Diab Vasc Dis Res 2011; 8:47-48.

31 Khalaf Ban H, Tuma Wafaa E, Hussein K I, Hussein Khalid R, Mohammad Haid R, Hussain Saad A: Thyroid function and other metabolic changes in married and unmarried females with polycystic ovary syndrome. Int Res J Pharm 2011;2:55-57.

32 Garelli S, Masiero S, Plebani M, Plebani M, Chen S, Furmaniak J, Armanini D, et al.. High prevalence of chronic thyroiditis in patients with polycystic ovary syndrome. Eur J Obstet Gynecol Reprod Biol. 2013;169:248-251.

33 Velija-Asimi Z: The role of subclinical hypothyroidism and vitamin D deficiency in the development of menstrual disorders in women with PCOS. Eur Soc Endocrinol 2012; 29:P916.

34 Benetti-Pinto CL, Berini Piccolo VR, Garmes HM, Juliato CR: Subclinical hypothyroidism in young women with polycystic ovary syndrome: an analysis of clinical, hormonal, and metabolic parameters. Fertil Steril 2013;99: 588-592.
35 Pei YJ, Wang AM, Zhao Y, Yan L, Li M, White $\mathrm{RE}$, et al: Studies of cardiovascular risk factors in polycystic ovary syndrome patients combined with subclinical hypothyroidism. Gynecol Endocrinol 2014;30:553-556.

36 Stroup DF, Berlin JA, Morton SC, Olkin I, Williamson GD, Rennie D, et al: Meta-analysis of observational studies in epidemiology: a proposal for reporting. Meta-analysis of observational studies in Epidemiology (MOOSE) group. J Am Med Assoc 2000;283: 2008-2012.

37 Bond CF, Wiitala WL, Dan Richard F: Metaanalysis of raw mean differences. Methods Psychol 2003;8:406-418.

38 Borenstein M, Hedges LV, Higgins JPT, Rothstein HR (eds). John Wiley, Introduction to Meta-Analysis. United Kingdom, 2009.

39 DerSimonrian R, Laird N: Meta-analysis in clinical trials. Control Clin Trials 1986;7:177188.

40 Sushma BJ, Nagarajappa K, Chandrakar S: A study on association of thyroid stimulating hormone with BMI, insulin resistance and lipid profile in women with polycystic ovarian syndrome. Int J Med Pharm Res 2014;2:585-590.

41 Pergialiotis V, Konstantopoulos P, Prodromidou A, Florou V, Papantaniou N, Perrea DN: The impact of subclinical hypothyroidism on anthropometric characteristics, lipid, glucose and hormonal profile of PCOS patients: a systematic review and meta- analysis. Eur J Endocrinol 2017;176:R159-R166.

42 Canaris GJ, Manowitz NR, Mayor G, Ridgway C: The Colorado thyroid disease prevalence study. Arch Int Med 2000;160:526-534.

43 Bakker SJ, Maaten JC, Popp-Snijders C, Slaets JPJ, Heine RJ, Gans RO: The relationship between thyrotropin and low density lipoprotein cholesterol is modified by insulin sensitivity in healthy euthyroid subjects. J Clin Endocrinol Metab 2001;86:1206-1211.
44 Laway BA, War FA, Shah S, Misgar RA, Kotwal SK: Alteration of lipid parameters in patients with subclinical hypothyroidism. Int J Endocrinol Metab 2014;12:e17496.

45 Cakir E, Sahin M, Topaloglu O, Colak NB, Karbek B, Gungunes A, et al: The relationship between LH and thyroid volume in patients with PCOS. J Ovarian Res 2012;5:43-48.

46 Liu XL, He S, Zhang SF, Wang J ,Sun XF, Gong CM, et al: Alteration of lipid profile in subclinical hypothyroidism: a meta-analysis. Med Sci Monit 2014;20:1432-1441.

47 Pappa T, Alevizaki M: Metformin and thyroid: an update. Eur Thyroid J 2013;2:22-28.

48 Morgante G, Musacchio MC, Orvieto R, Massaro MG, De Leo V: Alterations in thyroid function among the different polycystic ovary syndrome phenotypes. Gynecol Endocrinol 2013;29:967-969.

49 Dimitriadis G, Maratou E, Boutati E, Psarra K, Papasteriades C, Raptis SA: Evaluation of glucose transport and its regulation by insulin in human monocytes using flow cytometry. Cytometry A 2005;64:27-33.

50 Nakajima Y, Yamada M, Akuzawa M, Ishii S, Masamura Y, Satoh T, et al: Subclinical hypothyroidism and indices for metabolic syndrome in Japanese women: one-year followup study. J Clin Endocrinol Metab 2013;98: 3280-3287.

51 Bayram F, Boztosun A, Muderris I, Oner G: Effect of thyroid hormone replacement therapy on ovarian volume and androgen hormones in patients with untreated primary hypothyroidism. Ann Saud Med 2011;31.2: 145.

52 Shi X, Han C, Li C, Mao J, Wang W, Xie X, et al: Optimal and safe upper limits of iodine intake for early pregnancy in iodine-sufficient regions: a cross-sectional study of 7190 pregnant women in China. J Clin Endocrinol Metab 2015;100:1630-1638. 